\title{
Sequential and Site-Specific On-Surface Synthesis on a Bulk Insulator
}

\author{
Markus Kittelmann, ${ }^{\dagger}$ Markus Nimmrich, ${ }^{\dagger}$ Robert Lindner, ${ }^{\dagger}$ André Gourdon, ${ }^{\dagger}$ and Angelika Kühnle ${ }^{\dagger, *}$ \\ ${ }^{\dagger}$ Institut für Physikalische Chemie, Johannes Gutenberg-Universität Mainz, Duesbergweg 10-14, 55128 Mainz, Germany and ${ }^{\ddagger}$ CNRS, CEMES, Nanoscience Group, \\ 29 Rue J. Marvig, 31055 Toulouse, France
}

\begin{abstract}
The bottom-up construction of functional devices from molecular building blocks offers great potential in tailoring materials properties and functionality with utmost control. An important step toward exploiting bottom-up construction for real-life applications is the creation of covalently bonded structures that provide sufficient stability as well as superior charge transport properties over reversibly linked self-assembled structures. On-surface synthesis has emerged as a promising strategy for fabricating stable, covalently bound molecular structure on surfaces. So far, a majority of
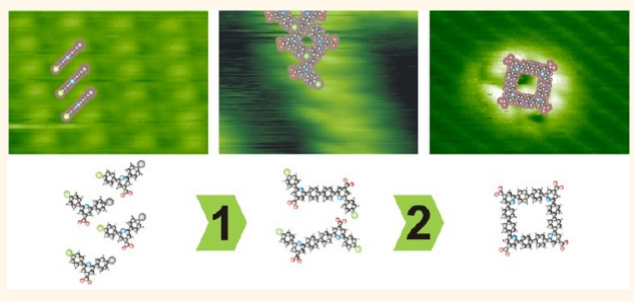
the structures created by this method have been obtained from a rather simple one-step processing approach. But the on-surface preparation of complex structures will require the possibility to carry out various reaction steps in a sequential manner as done in solution chemistry. Only one example exists in literature in which a hierarchical strategy is followed to enhance structural complexity and reliability on a metallic surface. Future molecular electronic application will, however, require transferring these strategies to nonconducting surfaces. Bulk insulating substrates are known to pose significant challenges to on-surface synthesis due to the absence of a metal catalyst and their low surface energy, frequently resulting in molecule desorption rather than reaction activation. By carefully selecting a suitable precursor molecule, we succeeded in performing a two-step linking reaction on a bulk insulating surface. Besides a firm anchoring toward the substrate surface, the reaction sites and sequential order are encoded in the molecular structure, providing so far unmatched reaction control in on-surface synthesis on a bulk insulating substrate.
\end{abstract}

KEYWORDS: on-surface synthesis $\cdot$ bulk insulating substrate $\cdot$ noncontact atomic force microscopy

$\mathrm{C}$ reating functional molecular structures on surfaces plays a central role in the field of nanotechnology, e.g., for the fabrication of future molecular electronic devices, ${ }^{1,2}$ molecular machines ${ }^{3}$ and novel materials with tailor-made properties. ${ }^{4}$ Molecular self-assembly provides a most powerful strategy for preparing functional molecular structures on supporting substrate surfaces. ${ }^{1,5}$ The selective interaction of purposively designed molecular building blocks has been tailored with great success to steer the resulting molecular structure in a well-controlled manner. ${ }^{5,6}$ To date, an impressive variety of selfassembled structures has been created in ultrahigh vacuum (UHV), ${ }^{7}$ ranging from perfect monolayer films ${ }^{8}$ over unidirectional rows ${ }^{9}$ and well-defined clusters to complex network structures. ${ }^{10}$

However, the inherent reversibility of the involved interactions limits the applicability of self-assembled structures in real-life environments. Consequently, strategies are required to fabricate functional structures that provide sufficient thermal and chemical stability for reliable operation at ambient conditions. Moreover, especially in the field of molecular electronics, improved charge transport properties are mandatory. Covalent linking of molecular precursors on the surface, referred to as on-surface synthesis, has recently emerged as a promising approach for the bottom-up fabrication of functional molecular structures with superior stability and increased electrical conductivity. ${ }^{11-14} \mathrm{~A}$ very exciting aspect of on-surface synthesis is the ability to follow new reaction pathways that might not be possible by classical solution synthesis. ${ }^{11}$ Only few examples for on-surface synthesis exist so far, demonstrating covalent linking on a supporting metal surface. ${ }^{13-27}$ Very recently, the concept of on-surface synthesis has been transferred to a thin insulating film of $\mathrm{NaCl}$ on $\mathrm{Ag}(100)^{28}$ and a bulk insulator, namely calcite $\left(\mathrm{CaCO}_{3}\right) \cdot{ }^{29}$ The latter studies are motivated by the need to decouple the electronic structure of the molecular device from the supporting surface
* Address correspondence to kuehnle@uni-mainz.de.

Received for review April 23, 2013 and accepted May 19, 2013.

Published online May 20, 2013 10.1021/nn402018w

C일 2013 American Chemical Society 
(a)
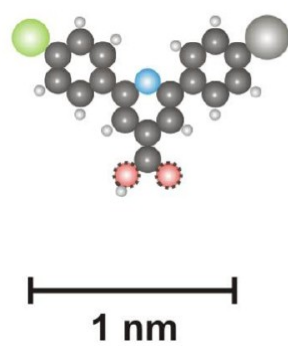

(b)

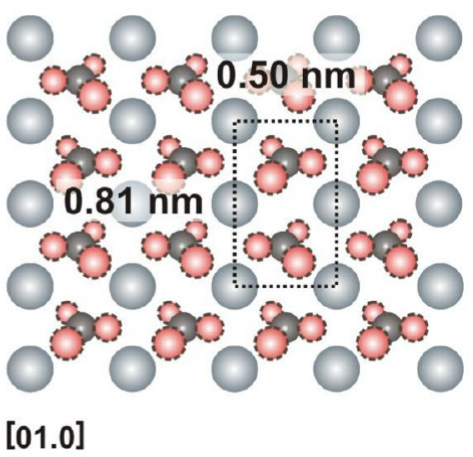

Figure 1. Model of (a) a 2-(4-bromophenyl)-6-(4-chlorophenyl)pyridine-4-carboxylic acid (BPCPPCA) molecule and (b) the calcite(10.4) surface. The $\mathrm{CaCO}_{3}(10.4)$ surface has a rectangular unit cell of size $0.50 \mathrm{~nm} \times 0.81 \mathrm{~nm}$, consisting of two carbonate groups and two calcium ions. The carbonate groups are rotated such that one oxygen atom lies above, one in and one below the plane spanned by the calcium ions.

when aiming at fabricating devices for future molecular electronics.

Up to now, the structural complexity of these structures has been very limited as most of the reactions have been performed in a single-step manner. Increasing the structural variety of on-surface synthesis requires utmost control with the ability to selectively induce sequential linking reactions in a hierarchical manner. This has recently been demonstrated using halide-substituted porphyrin derivatives on a $\mathrm{Au}(111)$ surface, $^{30,31}$ elegantly exploiting the specific dissociation energies of the bromine-phenyl and iodinephenyl bond. These dissociation energies are associated with different activation temperatures and thereby facilitate linking reactions in a site-specific and sequential manner. Thus, reaction sites and sequence are encoded in the structure of the precursor molecules. The latter results have, however, been obtained on a metallic substrate, limiting the applicability of these structures with regard to molecular electronics. So far, no example exists, demonstrating hierarchical control in on-surface synthesis on a bulk insulator substrate. Compared to metallic surfaces, bulk insulators are known to pose significant challenges when aiming at on-surface synthesis, especially when using thermal activation. Upon annealing, molecule desorption is frequently observed rather than reaction initiation. This can be explained by two aspects. First, the comparably low surface energy of insulating surfaces compared to metallic surfaces promotes desorption at relatively low temperatures. ${ }^{32}$ Second, in absence of a metallic surface known to act as a catalyst in Ullmanntype couplings, ${ }^{24}$ the activation step requires higher temperature on insulating surfaces.

Herein, we present the first successful example of improved structural control that is achieved from a site-specific and selective two-step linking process on a bulk insulator surface in UHV. The 2-(4-bromophenyl)6-(4-chlorophenyl)pyridine-4-carboxylic acid (BPCPPCA, Figure 1a) molecule used in this study comprises three functional groups. Bromophenyl and chlorophenyl groups were chosen for inducing site-specific and sequential covalent linking by homolytic cleavage of the halide-phenyl bonds due to their appropriate dissociation energies of 336 (Br-phenyl) and 399 (Cl-phenyl) $\mathrm{kJ} / \mathrm{mol}$, respectively. ${ }^{33}$ The third group is needed to provide sufficient anchoring toward the bulk insulator substrate, the natural cleavage plane of calcite $\left(\mathrm{CaCO}_{3}(10.4)\right.$, Figure $\left.1 \mathrm{~b}\right)$. A carboxylic acid moiety is known to bind sufficiently to this surface, ${ }^{29,34}$ which constitutes an inevitable prerequisite to allow for thermal activation of the desired reactions before the desorption temperature is reached. Moreover, we speculate that the binding of the deprotonated carboxylate group toward the surface facilitates cleavage of the halide-phenyl bond in the absence of a metal. ${ }^{29}$

When deposited onto the surface at room temperature, ordered islands are obtained as revealed by highresolution noncontact atomic force microscopy (NC-AFM). A first distinct change in molecular structure is induced upon annealing to $570 \mathrm{~K}$, which can be assigned to the homolytic cleavage of the brominephenyl bond and subsequent covalent linking of the resulting radicals, resulting in molecular dimers. Further annealing to $610 \mathrm{~K}$ induces the subsequent cleavage of the remaining chlorine-phenyl bond. The covalent coupling of the radicals now results in polymerization of the dimers into zigzag and closed ring structures, which are encoded by the specific design of the molecular building blocks. Our work demonstrates the successful application of a hierarchical two-step linking process on a bulk insulator surface, resulting in enhanced structural control that is programmed by the rational design of the molecular structure.

\section{RESULTS AND DISCUSSION}

Upon submonolayer deposition of BPCPPCA molecules onto the calcite surface held at room temperature, two types of ordered islands are observed as shown in the NC-AFM images in Figure 2. Overview 
images of island type $A$ and $B$ are given in Figure 2, panels $a$ and $b$, respectively. In Figure 2a, an A-type island is shown in the lower part of the image, while the upper darker area corresponds to the bare calcite surface. The island is composed of two different areas, displayed in darker and brighter color corresponding to an apparent height of 0.5 and $0.8 \mathrm{~nm}$, respectively. We stress, however, that height measurements need to be considered with care as force rather than topography is sensed in NC-AFM. The brighter area can be ascribed to a second layer occupation, which might be stabilized by intermolecular interactions between the basic pyridinic nitrogen atoms of the first layer molecules and the carboxylic acid functions of the second layer. Here, we focus on the darker area, which is ascribed to a monolayer of BPCPPCA molecules. Faint lines are visible that are oriented at an angle of $+50 \pm 3^{\circ}$

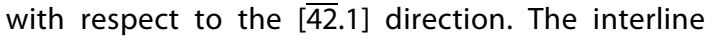
distance is approximately $1.2 \mathrm{~nm}$, compatible with the rows being composed of single molecules. A similar situation is found for the islands of type $B$ as shown in Figure $2 \mathrm{~b}$. An island with an apparent height of $0.5 \mathrm{~nm}$ is seen. As for island type $A$, areas with an apparent height of $0.8 \mathrm{~nm}$ are present that are tentatively assigned to a second molecular layer. Additionally, a few defects are observed in the monolayer film (lower right part of the image). The monolayer islands of type $B$ exhibit faint lines with a interline distance of $1.2 \mathrm{~nm}$ that are oriented at an

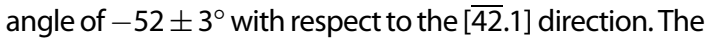
orientation of these lines indicates that the two island types represent the same molecular adsorption structure that is mirror-imaged along the [ $\overline{42.1]}$ axis. This assumption is further corroborated by the high-resolution images given in Figure $2 c$,d.

A high-resolution image of a type $A$ island is shown in Figure $2 c$. The faint lines can be resolved as individual double-lobe features (marked in Figure $2 c, d$ ) that arrange in rows. The periodic pattern is in agreement with a $(2 \times 4)$ superstructure as shown by the superimposed unit cell. A similar but mirrored situation is seen for the islands of type B as given in Figure $2 d$. Individual features are resolved that align in rows, resulting in a $(2 \times 4)$ superstructure. (An area with second-layer occupation is also seen in Figure $2 \mathrm{~d}$, which is not further discussed here.)

Based on these NC-AFM images, we can propose a tentative model of the molecular structure in the monolayer islands as discussed in Figure 3 . The $(2 \times 4)$ superstructure of island type $A(b)$ is shown in the upper (lower) part of Figure 3a. We ascribe each individually resolved feature to a single molecule. Based on the size of the molecules and the measured island height, we expect the molecules to be standing upright on the surface in a tilted fashion. Assuming that the molecules anchor toward the surface with the carboxylic acid anchor, the bright feature might be associated with a protruding halide atom. We note that
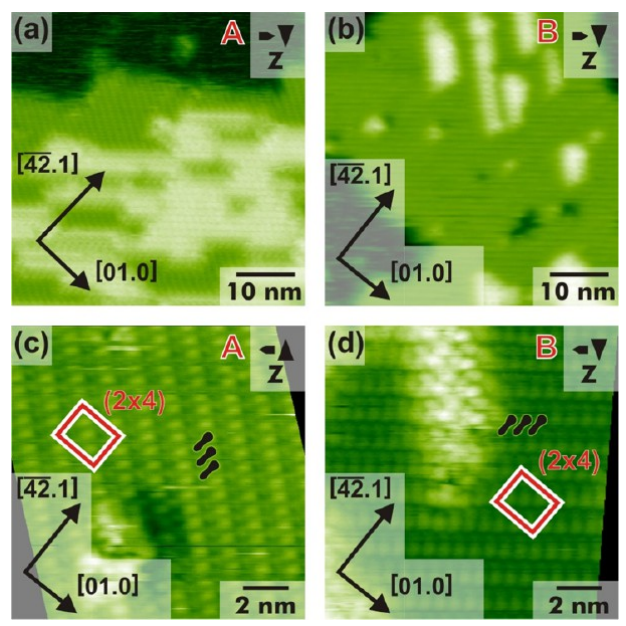

Figure 2. Molecular islands after deposition of BPCPPCA onto calcite(10.4) held at room temperature. (a) Island type A exhibiting faint lines oriented at an angle of $+50 \pm 3^{\circ}$ with respect to the [42.1] direction. A second-layer occupation is seen (areas with larger apparent height). (b) Island type B with faint lines oriented at an angle of $-52 \pm 3^{\circ}$ with respect

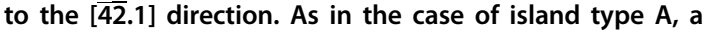
second layer occupation is revealed. (c) Drift-corrected high-resolution image of type $A$, revealing a $(2 \times 4)$ superstructure. Three molecules are marked by double-lobe features. (d) Drift-corrected high-resolution image of type $B$, confirming the same $(2 \times 4)$ molecular superstructure that is mirrored along the [ $\overline{4} \overline{2} .1]$ axis. Three molecules are marked by double-lobe features.

we only image one of the two halide atoms bright, which can be readily understood by a tilted arrangement and/or different interaction strength with the AFM tip, resulting in different apparent heights. A second less bright spot might be identified in the NC-AFM images that corresponds to the second halide atom. As shown by the ellipses indicating the approximate size of the molecule, our model fits excellently to the assumption of a $(2 \times 4)$ superstructure containing three molecules. The side views along the [01.0] and [42.1] directions are given in Figure $3 \mathrm{~b}$. We propose an anchoring of the carboxylic acid moiety toward the surface. Given the large negative shift of the contact potential of the islands with respect to the calcite surface (see Supporting Information), we assume that the carboxylic acid group is deprotonated. This assumption is in excellent agreement with the calculated $\mathrm{p} K_{\mathrm{a}}$ of the molecule ${ }^{35}$ of 3.5 and our previous studies revealing the deprotonation of a benzoic acid derivative. $^{36}$ The negatively charged carboxylate group is expected to anchor toward the positively charged surface calcium cations. We note, however, that the discussion made here does not depend on the protonation state of the carboxylic acid moiety. Along the [01.0] direction, the molecules are stacked in a shifted arrangement, which can be easily motivated by the optimization of $\pi-\pi$ interaction of the phenyl rings. It is known that $\pi-\pi$ interaction as well as $\pi$-halogen interaction favors a molecule-molecule distance of about $0.3 \mathrm{~nm} .{ }^{37}$ From this information, a tilt angle can 


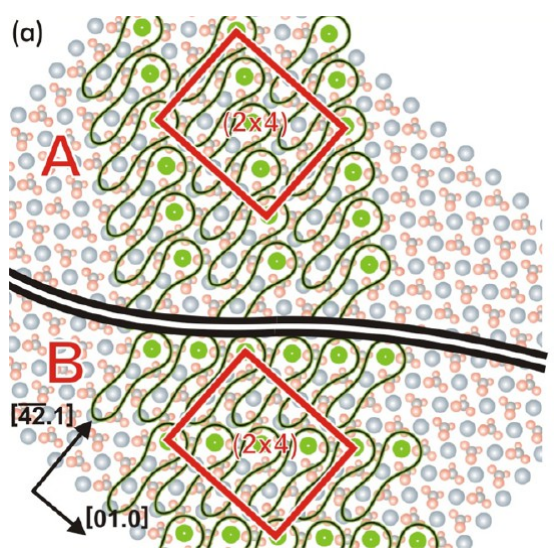

(b)
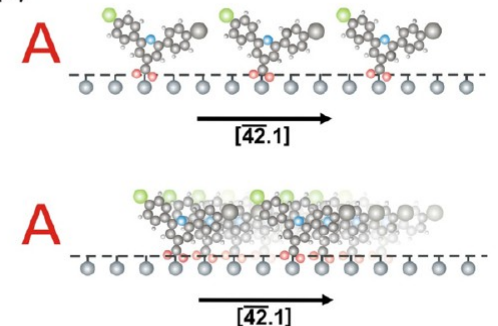

Figure 3. Tentative model for (a) the molecular arrangement of the molecules in type A and B islands. The structures are

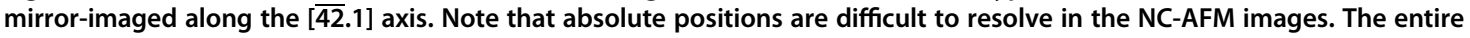
structure can, thus, be shifted with respect to the surface. (b) Side view of the proposed arrangement of the molecules within island $A$. This model is based on the assumption that the molecule is anchored toward the surface with the negatively charged carboxylate group positioned above a surface calcium cation. The structure for the molecules within island type $B$ is the same as island $A$, but the mirror image.

be estimated, as tilting of the molecule results in a different molecule-molecule distance. To ensure a molecule-molecule distance of $0.3 \mathrm{~nm}$, a tilt angle of $35^{\circ}$ is assumed. Although height measurements in NC-AFM must not be taken literally, we note that the apparent height of $0.5 \mathrm{~nm}$ is in good agreement with this tilted arrangement.

The situation as revealed after deposition onto the surface held at room temperature changes drastically upon annealing the substrate to $570 \mathrm{~K}$, indicating a significant change in the molecular structure. A representative NC-AFM is given in Figure 4a. Instead of ordered islands, row-like structures are formed on the surface. Although the overall ordering is poor compared to the islands, two main orientations of the rowlike structures (marked with I and II) can be identified. The two orientations span an angle of approximately $49 \pm 3^{\circ}$. Closer imaging of the row structure of type ll as shown in Figure $4 \mathrm{~b}$ reveals a periodic structure within the rows. The same holds true for the rows of type I as shown in Figure 4c. The spanning angle of $49^{\circ}$ can be obtained by considering an angle of $+64 \pm 3^{\circ}$ and $-67 \pm 3^{\circ}$ with respect to the $[\overline{42} .1]$ direction for the I and II rows. Thus, this angle and the similarity of the two row structures indicate that these two structures are, in fact, identical but mirror images of each other.

This indicates that the envisioned linking reaction has taken place upon homolytic cleavage of the bromide atoms. A dimer structure that is covalently linked at the former bromine position $\left(6,6^{\prime}-\left(\left[1,1^{\prime}\right.\right.\right.$-biphenyl]4,4'-diyl)bis(2-(4-chlorophenyl)isonicotinic acid)) exhibits either a $\mathrm{U}$ or an $\mathrm{S}$-like conformation. We assume that the dimer molecules still anchor with the two carboxylate groups toward the surface calcium cations. This is equally possible for both, the $U$ and the $S$ form (see Supporting Information). It is expected that the dimers pack to allow for intermolecular interaction. The observed $1.1 \mathrm{~nm}$ periodicity along the rows agrees
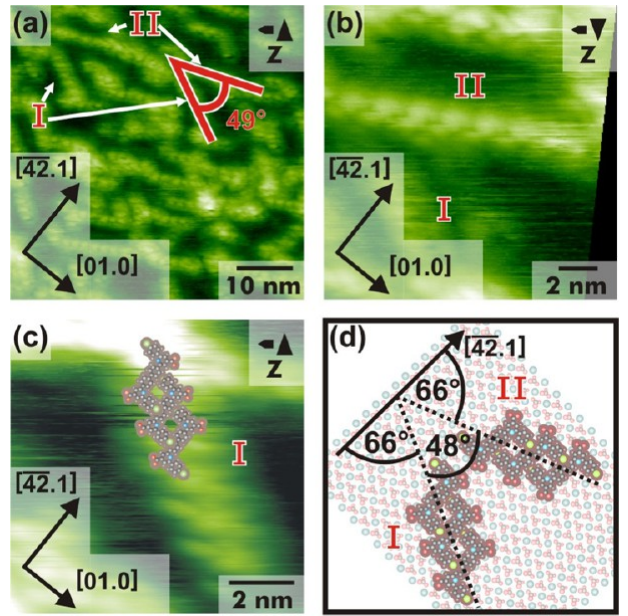

Figure 4. Distinctly different structure obtained after annealing the substrate to about $570 \mathrm{~K}$ for $1 \mathrm{~h}$. (a) Overview image, revealing row-like structures forming two types (I and II). (b) Drift-corrected, detailed image of row type II. (c) Drift-corrected, detailed image of row type I. The individual features within the rows fit in size with dimer molecules as can seen by the superimposed dimer models. (d) Proposed model for the two different row types on calcite(10.4).

with the proposed packing of the S-type molecules, as superimposed in the NC-AFM image given in Figure 4c. An optimization of the intermolecular distance in order to allow for halogen- $\pi$ interaction can be understood as the driving force behind the lateral shift of the molecules perpendicular to the row direction. This readily results in a molecular row that forms an angle

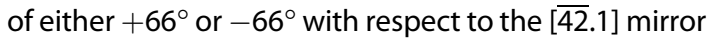
axis, in excellent agreement with the experimentally obtained opening angle between the $\pm 66^{\circ}$-rows of $48^{\circ}$ (see Figure $4 \mathrm{~d}$ ). This angle provides a second, independent observation that supports the model proposed here.

We thus conclude this part by summarizing that significantly different structures are obtained upon 

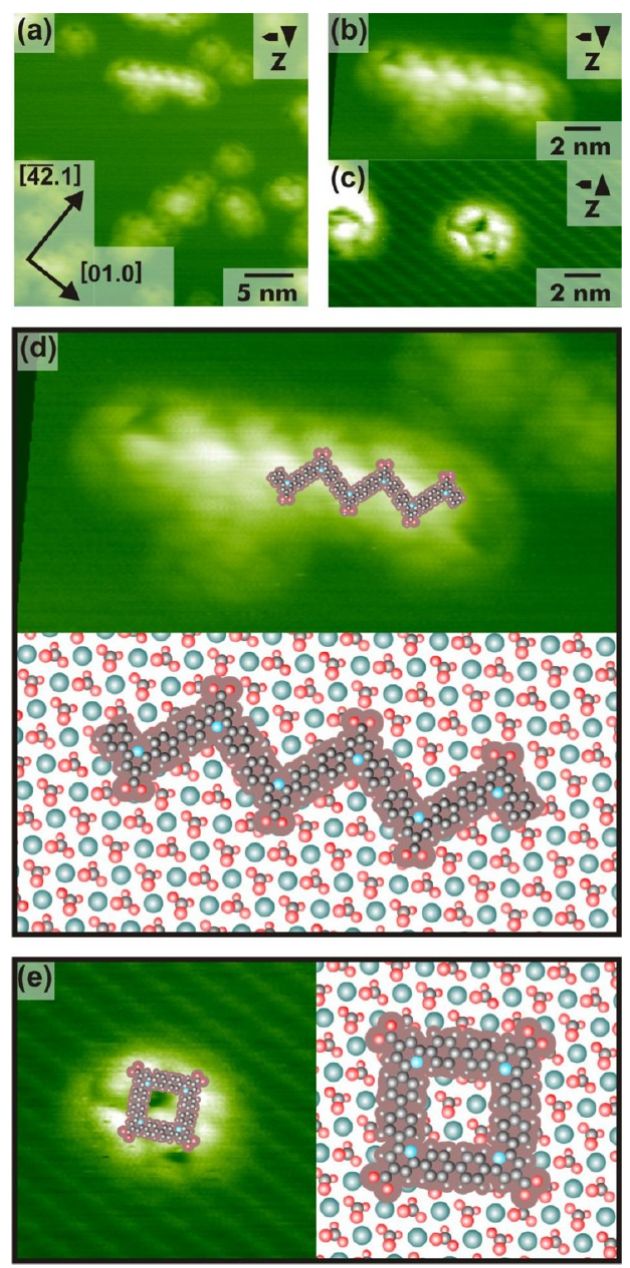

Figure 5. Structures observed after the second annealing step at $610 \mathrm{~K}$ for $1 \mathrm{~h}$. (a) Chains oriented along an angle of $-60 \pm 3^{\circ}$ with respect to the [42.1] direction and ring-like features are observed to coexist on the surface. (b) Highresolution image of a chain structure. (c) High-resolution image of a closed ring structure. Note that the rows running from the upper left to lower right originate from the calcite(10.4) substrate. (d) Drift-corrected image of the chain structure and proposed model. (e) Drift-corrected image of the ring structure with proposed model.

moderate annealing. The individual features within the rows fit in size with dimer molecules. Moreover, the observed features can be readily explained by a rational assembly of the dimers. All three findings agree well with the assignment of a first covalent reaction step involving the cleavage of the bromine-phenyl bond.

Next, we anneal the surface with the row structures in a second step at $610 \mathrm{~K}$. The resulting structures are shown in Figure 5. Most strikingly, the molecular structures that are present on the surface after the second annealing steps are, again, distinctly different from both, the as-deposited ordered islands and the row structures observed after the first, more moderate annealing step. Now, chains oriented along an angle of $-60 \pm 3^{\circ}$ with respect to the [42.1] direction and ringlike features are observed to coexist on the surface
(Figure 5a). The chains (Figure 5b) differ in appearance, periodicity and, most notably, in orientation with respect to the underlying substrate lattice, clearly indicating that the chains are composed of other building blocks than the row structures revealed after moderate annealing. Moreover, ring-like features (Figure $5 c$ ) are seen on the surface, exhibiting a distinct internal structure. To elucidate the structural details, we first consider the chain structure as shown in Figure $5 \mathrm{~d}$. A periodic structure of $1.7 \mathrm{~nm}$ is clearly resolved that fits excellently in size with a structure that is composed by further linking of the dimer molecules via cleavage of the chlorine-phenyl bond. Arranging the model such that the carboxylate groups can bind toward the calcium cations results in an angle of $-61^{\circ}$ with respect to the [42.1] direction, exactly matching the experimentally observed results. Thus, the size match, the agreement of the periodic repeat distance and the observed orientation on the surface corroborate the model of a further linking of the dimer units in a zigzag polymer. A closer look at the dimer units reveals that a second structure is feasible when linking the dimer building blocks to a closed tetramer ring, which is observed experimentally. The ring tension in these large cyclic tetramers is distributed over twelve aromatic rings and can be considered as negligible. We have not observed larger rings which might be explained by two effects. First, when two dimers meet, a cyclic tetramer might form before a third dimer arrives. Second, the likelihood for ring closure is expected to decrease with increasing number of dimers as the distance of the reactive ends increases. A detailed analysis as well as can be seen in Figure 5e confirms the excellent size match. Moreover, the internal structure seen as dark stripes in the ring-like feature suggests that the four initial precursor molecules are resolved. We assign the bright part to the carboxylate group that is again positioned atop a surface calcium atom. We note that three out of the four carboxylate groups can attain perfect on-top positions while the forth group does not fit to the surface lattice. This size mismatch of the ring structure with the underlying calcite lattice might explain why one of the four bright features is imaged in a different way compared to the three other bright features within a ring.

To summarize the results of the second annealing step, we note that yet different structures are obtained after the second activation step. Two coexisting structures are revealed, as expected from the structure of the molecular precursors. Extended rows can be identified that fit in size, periodicity and orientation to the model of a further linked zigzag polymer. Linking two dimers in a closed fashion results in ring-like features that are observed to coexist on the surface. The sum of these experimental findings strongly indicates the successful activation of the second reaction step involving the chlorine-phenyl bond. 


\section{CONCLUSION}

In conclusion, we present the first successful example of a two-step polymerization reaction on the surface of a bulk insulator. Carefully selecting a precursor molecule that provides both the encoding of the selective and sequential reaction sites as well as suitable anchor groups for preventing desorption is demonstrated to result in hierarchical polymerization. Extended zigzag and closed ring structures are created upon sequential activation of homolytic cleavage of first bromine-phenyl and second chlorine-phenyl bonds. This work demonstrates that enhanced structural control in on-surface synthesis can be achieved even on the weakly interacting surface of a bulk insulator.

\section{METHODS}

Calcite Preparation. Optical quality calcite samples from Korth Kristalle GmbH (Kiel, Germany) are cleaved in situ, resulting in flat (10.4) cleavage planes. ${ }^{38}$ Right after cleavage, the crystals are annealed at $600 \mathrm{~K}$ for about $1 \mathrm{~h}$ to remove surface charges.

Molecule Deposition. The molecules (97\% purity) are purchased from Sigma-Aldrich Chemie GmbH (Taufkirchen, Germany) and thoroughly outgassed at $310 \mathrm{~K}$ for $40 \mathrm{~h}$ prior to use. The molecules are sublimated in situ onto the freshly prepared calcite surface held at room temperature using a home-built Knudsen cell. The images shown here are achieved after sublimation for $15 \mathrm{~min}$ with a cell temperature of $336 \mathrm{~K}$, corresponding to a flux of approximately 0.03 monolayers $/ \mathrm{min}$.

NC-AFM Imaging. All experiments are carried out under UHV conditions (base pressure $\leq 1 \times 10^{-10} \mathrm{mbar}$ ) using a VT AFM 25 atomic force microscope (Omicron, Taunusstein, Germany) operated in the frequency modulation noncontact mode (NC-AFM). The system is equipped with an easyPLL Plus controller and phase-locked loop detector (Nanosurf, Liestal, Switzerland) for oscillation excitation and signal demodulation. We use n-doped silicon cantilevers (NanoWorld, Neuchâtel, Switzerland) with resonance frequencies of around $300 \mathrm{kHz}$ (type PPP-NCH) excited to oscillation amplitudes of about $10 \mathrm{~nm}$. Prior to their use, the cantilevers were $\mathrm{Ar}^{+}$sputtered at $2 \mathrm{keV}$ for $5 \mathrm{~min}$ to remove contaminants. Image type as well as fast and slow scan directions are given in the upper right corner of each image.

Thermal Activation Step. The molecules are thermally activated by annealing the calcite substrate with a pyrolytic boron nitride heater positioned underneath the sample. The temperature is controlled by a thermocouple mounted at the sample stage about $2.5 \mathrm{~cm}$ apart from the sample. The temperature specifications given in this work are the corresponding temperatures expected at the calcite sample based on an individual calibration curve supplied by the manufacturer (Omicron, Taunusstein, Germany).

Conflict of Interest: The authors declare no competing financial interest.

Acknowledgment. Financial support from the German Research Foundation through grant DFG-ANR Project KU 1980/5-1 is gratefully acknowledged.

Supporting Information Available: KPFM data for BPCPPCA molecules on calcite at room temperature and proposed adsorption positions for $\mathrm{U}$ form and $\mathrm{S}$ form. This material is available free of charge via the Internet at http://pubs.acs.org.

\section{REFERENCES AND NOTES}

1. Joachim, C.; Gimzewski, J. K.; Aviram, A. Electronics Using Hybrid-Molecular and Mono-Molecular Devices. Nature 2000, 408, 541-548.

2. Barth, J. V.; Costantini, G.; Kern, K. Engineering Atomic and Molecular Nanostructures at Surfaces. Nature 2005, 437, 671-679.

3. Kay, E. R.; Leigh, D. A.; Zerbetto, F. Synthetic molecular motors and mechanical machines. Angew. Chem., Int. Ed. 2007, 46, 72.

4. Whitesides, G. M.; Mathias, J. P.; Seto, C. T. Molecular SelfAssembly and Nanochemistry: A Chemical Strategy for the
Synthesis of Nanostructures. Science 1991, 254, 13121319.

5. Barth, J. V. Molecular Architectonic on Metal Surfaces. Annu. Rev. Phys. Chem. 2007, 58, 375-407.

6. Slater, A. G.; Beton, P. H.; Champness, N. R. TwoDimensional Supramolecular Chemistry on Surfaces. Chem. Sci. 2011, 2, 1440-1448.

7. Yokoyama, T.; Yokoyama, S.; Kamikado, T.; Okuno, Y.; Mashiko, S. Selective Assembly on a Surface of Supramolecular Aggregates with Controlled Size and Shape. Nature 2001, 413, 619-621.

8. Schreiber, F. Structure and Growth of Self-Assembling Monolayers. Prog. Surf. Sci. 2000, 65, 151-256.

9. Böhringer, M.; Morgenstern, K.; Schneider, W.-D.; Berndt, R.; Mauri, F.; Vita, A. D.; Car, R. Two-Dimensional SelfAssembly of Supramolecular Clusters and Chains. Phys. Rev. Lett. 1999, 83, 324-327.

10. Pawin, G.; Wong, K. L.; Kwon, K. Y.; Bartels, L. A Homomolecular Porous Network at a $\mathrm{Cu}(111)$ Surface. Science 2006, 313, 961-962.

11. Franc, G.; Gourdon, A., Covalent Networks through on-Surface Chemistry in Ultra-High Vacuum: State-of-the-Art and Recent Developments. Phys. Chem. Chem. Phys. 2011, online.

12. Gourdon, A. On-Surface Covalent Coupling in Ultrahigh Vacuum. Angew. Chem., Int. Ed. 2008, 47, 6950-6953.

13. Grill, L.; Dyer, M.; Lafferentz, L.; Persson, M.; Peters, M. V.; Hecht, S. Nano-Architectures by Covalent Assembly of Molecular Building Blocks. Nat. Nanotechnol. 2007, 2, 687-691.

14. Cai, J. M.; Ruffieux, P.; Jaafar, R.; Bieri, M.; Braun, T.; Blankenburg, S.; Muoth, M.; Seitsonen, A. P.; Saleh, M.; Feng, X. L.; et al. Atomically Precise Bottom-up Fabrication of Graphene Nanoribbons. Nature 2010, 466, 470-473.

15. Bartels, L. Tailoring Molecular Layers at Metal Surfaces. Nat. Chem. 2010, 2, 87-95.

16. Weigelt, S.; Bombis, C.; Busse, C.; Knudsen, M. M.; Gothelf, K. V.; Lægsgaard, E.; Besenbacher, F.; Linderoth, T. R. Molecular Self-Assembly from Building Blocks Synthesized on a Surface in Ultrahigh Vacuum: Kinetic Control and Topo-Chemical Reactions. ACS Nano 2008, 2, 651-660.

17. Weigelt, S.; Busse, C.; Bombis, C.; Knudsen, M. M.; Gothelf, K. V.; Lægsgaard, E.; Besenbacher, F.; Linderoth, T. R. Surface Synthesis of $2 \mathrm{~d}$ Branched Polymer Nanostructures. Angew. Chem., Int. Ed. 2008, 47, 4406-4410.

18. Weigelt, S.; Busse, C.; Bombis, C.; Knudsen, M. M.; Gothelf, K. V.; Strunskus, T.; Wöll, C.; Dahlbom, M.; Hammer, B.; Lægsgaard, E.; et al. Covalent Interlinking of an Aldehyde and an Amine on a Au(111) Surface in Ultrahigh Vacuum. Angew. Chem., Int. Ed. 2007, 46, 9227-9230.

19. Matena, M.; Riehm, T.; Stöhr, M.; Jung, T. A.; Gade, L. H. Transforming Surface Coordination Polymers into Covalent Surface Polymers: Linked Polycondensed Aromatics through Oligomerization of N-Heterocyclic Carbene Intermediates. Angew. Chem., Int. Ed. 2008, 47, 2414-2417.

20. Veld, M. I.; lavicoli, P.; Haq, S.; Amabilino, D. B.; Raval, R. Unique Intermolecular Reaction of Simple Porphyrins at a Metal Surface Gives Covalent Nanostructures. Chem. Commun. 2008, 1536-1538.

21. Zwaneveld, N. A. A.; Pawlak, R.; Abel, M.; Catalin, D.; Gigmes, D.; Bertin, D.; Porte, L. Organized Formation of 2d Extended Covalent Organic Frameworks at Surfaces. J. Am. Chem. Soc. 2008, 130, 6678-6679. 
22. Gutzler, R.; Walch, H.; Eder, G.; Kloft, S.; Heckl, W. M.; Lackinger, M. Surface Mediated Synthesis of $2 d$ Covalent Organic Frameworks: 1,3,5-Tris(4-bromophenyl)benzene on Graphite(001), $\mathrm{Cu}(111)$, and $\mathrm{Ag}(110)$. Chem. Commun. 2009, 4456-4458.

23. Perepichka, D. F.; Rosei, F. Chemistry Extending Polymer Conjugation into the Second Dimension. Science 2009, $323,216-217$.

24. Lipton-Duffin, J. A.; Ivasenko, O.; Perepichka, D. F.; Rosei, F. Synthesis of Polyphenylene Molecular Wires by SurfaceConfined Polymerization. Small 2009, 5, 592-597.

25. Treier, M.; Richardson, N. V.; Fasel, R. Fabrication of SurfaceSupported Low-Dimensional Polyimide Networks. J. Am. Chem. Soc. 2008, 130, 14054-14055.

26. Treier, M.; Ruffieux, P.; Gröning, P.; Xiao, S. X.; Nuckolls, C.; Fasel, R. An Aromatic Coupling Motif for Two-Dimensional Supramolecular Architectures. Chem. Commun. 2008, 4555-4557.

27. Bombis, C.; Ample, F.; Lafferentz, L.; Yu, H.; Hecht, S.; Joachim, C.; Grill, L. Single Molecular Wires Connecting Metallic and Insulating Surface Areas. Angew. Chem., Int. Ed. 2009, 48, 9966-9970.

28. Abel, M.; Clair, S.; Ourdjini, O.; Mossoyan, M.; Porte, L. Single Layer of Polymeric Fe-Phthalocyanine: An Organometallic Sheet on Metal and Thin Insulating Film. J. Am. Chem. Soc. 2011, 133, 1203-1205.

29. Kittelmann, M.; Rahe, P.; Nimmrich, M.; Hauke, C. M.; Gourdon, A.; Kühnle, A. On-Surface Covalent Linking of Organic Building Blocks on a Bulk Insulator. ACS Nano 2011, 5, 8420-8425.

30. Lafferentz, L.; Eberhardt, V.; Dri, C.; Africh, C.; Comelli, G.; Esch, F.; Hecht, S.; Grill, L. Controlling on-Surface Polymerization by Hierarchical and Substrate-Directed Growth. Nat. Chem. 2012, 4, 215-220.

31. Faury, T.; Clair, S.; Abel, M.; Dumur, F.; Gigmes, D.; Porte, L. Sequential Linking To Control Growth of a Surface Covalent Organic Framework. J. Phys. Chem. C. 2012, 116, 48194823.

32. Rahe, P.; Lindner, R.; Kittelmann, M.; Nimmrich, M.; Kühnle, A. From Dewetting to Wetting Molecular Layers: $C_{60}$ on $\mathrm{CaCO}_{3}(10-14)$ as a Case Study. Phys. Chem. Chem. Phys. 2012, 14, 6544-6548.

33. Luo, Y.-R. Comprehensive Handbook of Chemical Bond Energies; CRC Press: Boca Raton, FL, 2007.

34. Duffy, D. M.; Harding, J. H. Modelling the Interfaces between Calcite Crystals and Langmuir Monolayers. J. Mater. Chem. 2002, 12, 3419-3425.

35. Chemical Abstracts, calculated using Advanced Chemistry Development (ACD/Labs) Software V11.02 (1994-2013 ACD/Labs).

36. Kittelmann, M.; Rahe, P.; Gourdon, A.; Kühnle, A. Direct Visualization of Molecule Deprotonation on an Insulating Surface. ACS Nano 2012, 8, 7411.

37. Prasanna, M. D.; Guru Row, T. N. C-Halogen ... I Interactions and Their Influence on Molecular Conformation and Crystal Packing: A Database Study. Cryst. Eng. 2000, 3, 135-154.

38. Tröger, L.; Schütte, J.; Ostendorf, F.; Kühnle, A.; Reichling, M. Concept for Support and Cleavage of Brittle Crystals. Rev. Sci. Instrum. 2009, 80, 063703. 